\title{
90. On the Order of the Absolute Values of a Linear Form, (Third Report.)
}

\author{
By Seigo Fukazawa.
}

Tokyo Butsuri Gakko.

(Rec. May 31, 1927. Comm. by M. FujrwarA, M.I.A., June 12, 1927.)

1. In two Notes published in these Proceedings ${ }^{1)}$ I have reported on my researches on the order of $|a x-y+\beta|$. Especially, in the second of these notes I have given the upper limit of $\lim \inf |x(\alpha x-y+\beta)|$ for some classes of the irrational number $\alpha$. Since then I have got for some cases the more precise and best possible limit, which will be shown in the following lines.

In the case iii l.c. that is, when $\lim \inf q_{i}=2 k$, we have the following theorem :

If $k \geqq 3$ and $a x-y-\beta$ is not equivalent to

$$
\sqrt{k^{2}+1} x-y+\frac{\sqrt{k^{2}+1}-k+1}{2} \text {, }
$$

then

$$
\lim \inf |x(\alpha x-y+\beta)| \leqq \frac{1}{4\left\{\frac{1}{1+\omega}+\frac{1}{2 k-1}+\frac{1}{2 k+\omega}\right\}},
$$

where

$$
\begin{aligned}
\omega & =\frac{1}{2 k+2}+\frac{1}{2 k+4}+\frac{1}{2 k+2}+\frac{1}{2 k+4}+\cdots \cdots, & & \text { if } k \geqq 6 \\
& =\frac{1}{2 k+2}+\frac{1}{2 k+6}+\frac{1}{2 k+2}+\frac{1}{2 k+6}+\cdots \cdots, & & \text { if } k=4,5 \\
& =\frac{1}{8}+\frac{1}{16}+\frac{1}{8}+\frac{1}{16}+\cdots \cdots, & & \text { if } k=3 .
\end{aligned}
$$

The equality in (A) occurs for infinitely many (non-enumerable) forms, and if $\varepsilon$ is any positive number smaller than the right-hand side

1) On the extension of Klein's geometrical interpretation of continued fraction, these Proc. 2, 100. On the extension of a theorem of Minkowski, ibid. 2, 305. 
of (A), then there are infinitely many (non-enumerable) forms, for which

$$
\lim \inf q_{i}=2 k,
$$

and

$$
\lim \inf |x(\alpha x-y+\beta)|=\varepsilon \text {. }
$$

In the case iv, that is, when $q_{i}=2 k$ and $\mu_{i}=1$ or $\mu_{i+1}=1$ for infinitely many $i$, we have

$$
\lim \inf |x(\alpha x-y+\beta)| \leqq \frac{1}{4\left(1+\frac{1}{2 k-1}+\frac{2}{2 k}\right)} .
$$

The equality in B occurs for infinitely many (non-enumerable) forms, and if $\varepsilon$ is any positive number smaller than the right-hand side of (B), then there exist also infinitely many (non-enumerable) forms with the character above-mentioned, for which

$$
\lim \inf \left|x^{\prime}(\alpha x-y+\beta)\right|=\varepsilon .
$$

2. Khintchine, in his paper " Uber eine Klasse linearer diophantischer Approximation," has given some theorems on the order of $|a x-y|$ and $|a x-y+\beta|$. We can prove these theorems by means of our method. Especially his theorem IV - "There exists an absolute constant $\gamma$ with the following character : for any real number $\alpha$ we can determine $\beta$, so that the inequality $|a x-y+\beta|<\frac{\gamma}{x}$ has no solution for integral values of $x>0$ and $y$ "-can be proved more simply than by his method, and moreover our method gives the precise result

$$
r>\frac{1}{457}
$$

We have also the theorem :

"For any real number $\alpha$ we can determine $\beta$, such that

$$
\lim \inf |x(\alpha x-y+\beta)|=0 . "
$$

1) Rend. Parelmo, 50, (1927). 